Abstract PTU-195 Table 1 Gluten sensitive symptoms reported in the adult community

\begin{tabular}{|ll|}
\hline Intestinal & Extra-intestinal \\
\hline Bloating 78\% & Fatigue 23\% \\
Abdominal discomfort 38\% & Headaches 22\% \\
Abdominal pain 29\% & Joint pains $8 \%$ \\
Constipation $21 \%$ & Rash $6 \%$ \\
Diarrhoea 16\% & Numbness $6 \%$ \\
Flatulence $14 \%$ & Confusion $5 \%$ \\
Nausea $9 \%$ & \\
\hline
\end{tabular}

Conclusion $13 \%$ of the general population self report GS. Of those investigated in the gastroenterology department only $10 \%$ have CD, with the remaining fulfilling the criteria for NCGS. The symptoms of NCGS are diverse and there is a relationship with IBS. The prevalence of a positive HLA typing in NCGS is half that of CD suggesting an alternative immune mechanism to gluten intolerance.

Disclosure of Interest None Declared.

\section{PTU-196 SMALL BOWEL CAPSULE ENDOSCOPY - A RETROSPECTIVE ANALYSIS OF 348 CASES FROM OEII AND LISTER HOSPITALS}

doi:10.1136/gutjnl-2013-304907.286

1."J A Evans, 'S Beg, 'R Nathwani, 'K Samsheer, 'E Ebro, 'E Turnbull. 'Department of Gastroenterology, Queen Elizabeth II \& Lister Hospitals, East \& North Herts NHS Trust, UK

Introduction Small bowel capsule endoscopy (SBCE) is a useful diagnostic tool for investigating iron deficiency anaemia (IDA) and suspected Crohn's disease (CD) ${ }^{1,2}$, but its use is often limited to specialist teaching centres. We aim to establish whether SBCE is a useful tool when employed in a district general hospital (DGH) setting.

Methods We retrospectively analysed the data of all SBCE carried out at QEII and Lister hospitals over 36 months since its introduction in 2009 using the GIVEN Pillcam SB 2 system. Patient demographics, indications, previous investigations, SBCE findings and how these altered management were reviewed.

Results 348 studies were performed on 175 males and 173 females; the mean age was 54.3 years (range 17-86). 207/348 (59\%) revealed some form of pathology. 7 studies were incomplete (2.01\%). Patency capsules were used in those with suspected risk of stricturing pathology for $22 / 348$ cases $(6.32 \%)$. No cases required surgical retrieval of a capsule.

The most common indication for SBCE was IDA = 185/348 studies $(53.1 \%)$. The next most common indication was suspected $\mathrm{CD}=141 / 348(40.5 \%)$. All patients had undergone bi-directional endoscopy prior to SBCE.

The commonest findings were; small bowel (SB) erosions/ ulcers (65 cases) SB angiodysplasia (39 cases) CD (21 cases) SB ulcers (19) polypoidal masses (13 cases) and fresh blood (11 cases). 3 cases of lymphoma and one of Enterobius vermicularis were discovered.

Where IDA was the indication, 65/185 studies (35.1\%) had findings that led to an alteration in management. In studies for suspected CD, 44/141 (31.2\%) had findings that led to an alteration in management.

Conclusion For IDA the diagnostic yield of 35\% is in keeping with previously published data, which shows that SBCE can identify a source of bleeding in $31-76 \% 1$.

For $\mathrm{CD}$ we identified abnormal pathology requiring further intervention in $31 \%$ of cases. It is debatable whether a small number of terminal ileal ulcers is sufficient to diagnose CD. If greater than 3 are seen, histological confirmation of $\mathrm{CD}$ is attempted via repeat endoscopy.

9 of the 348 patients had previously undergone a SBCE and the less invasive nature of SBCE lends itself to repeated use for small bowel CD surveillance.

SBCE is a reliable, safe and useful tool in the setting of a DGH setting for identifying small bowel pathology and should not be restricted to specialist centres.

Disclosure of Interest None Declared.

\section{REFERENCES}

R Sidhu, D Sanders, A Morris, M McAlindon. Guidelines on small bowel enteroscopy and capsule endoscopy in adults. BSG Guidelines. Gut 2008; 57:125-136.

SK Patel, P Mairs. A Review of Small Bowel Capsule Endoscopy Performed At Darent Valley Hospital Since Its Introduction in 2006. The Online Journal of Clinical Audits. 2011; Vol 3(3)

\section{PTU-197 A PATIENT SATISFACTION SURVEY FOR COELIAC DISEASE IN LEEDS - WHAT DO PATIENTS WANT?}

doi:10.1136/gutjnl-2013-304907.287

1."J S R Jennings. 'Gastroenterology, Leeds Teaching Hospitals NHS Trust, Leeds, UK

Introduction The long term follow up for patients with Coeliac disease is controversial. There are a number of models used in the UK which include follow up by primary care, conventional gastroenterology clinics, specialist Coeliac clinics \& dietician- or nurse-led services. National guidelines do not directly address this issue. I developed a patient satisfaction questionnaire with the Leeds local group of Coeliac UK to survey opinion.

Methods A 30 point patient directed questionnaire was sent electronically to members of the Leeds Coeliac UK group. The questionnaire asked for basic demographics, details of diagnosis, compliance with the gluten free diet (GFD), opinions on the service provided \& asked for a preference for the long term follow up.

Results 630 patients were sent an email copy of the questionnaire. I received 137 completed replies (22\%). Mean age 61 years (range 8-91), 78\% female, 96\% British Caucasian.

$18 \%$ had been diagnosed by their GP, 37\% a non-specified consultant, 35\% Gastroenterologist, 5\% colorectal surgeon, $6 \%$ Paediatrics \& $3 \%$ self diagnosed. Mean age at diagnosis was 48 (range 0.5 to 78 ) with a mean of 6 years from onset of symptoms to diagnosis.

$77 \%$ claimed to be strict with the GFD, 21\% described lapses with $2 \%$ variably compliant. $65 \%$ did not eat oats.

The majority (77\%) wished to be monitored long term. Currently $45 \%$ saw their GP only, 29\% attend general Gastroenterology clinics \& 26\% a Specialist Coeliac clinic. $82 \%$ described clinics as useful. In the future $28 \%$ preferred follow up to be provided by a GP with guidelines, $5 \%$ a general clinic, $60 \%$ a Coeliac clinic \& $5 \%$ a dietician-led service. $2 \%$ wanted no follow up. $68 \%$ wished to be seen yearly, $10 \% 2$ yearly.

Patients described their experience of in-patient care episodes as poor. $68 \%$ described staff knowledge of the GFD as average or poor, $87 \%$ had average or poor access to a GFD \& $73 \%$ described poor access to dietetic services on the ward. $83 \%$ described cafe or canteen services for a GFD as poor.

$59 \%$ had not received Pnemovax with $4 \%$ unsure.

Conclusion This is a large patient survey. The responders are clearly a self-selecting group. Even so a third prefer long term follow up with their GP, with the majority asking for specialist care. The poor number of Pneumovax recipients suggests guidelines are needed for primary care. This data may help guide the future development of services. Perhaps the time has come for a national survey. The experience of patients with Coeliac disease in hospital is a concern.

Disclosure of Interest None Declared 